\title{
Hematology and blood biochemistry in wild hybrid marmosets from the Atlantic Forest, Brazil
}

\author{
Hematologia e bioquímica sanguínea de saguis selvagens híbridos da \\ Mata Atlântica, Brasil
}

\begin{abstract}
Ita de Oliveira e Silva ${ }^{I}$ Fernanda de Fátima Rodrigues da SilvaII Lisieux Franco Fuzessy ${ }^{\text {III }}$ Alexandre de Oliveira Tavela ${ }^{\mathrm{IV}}$ Moacir Carretta JúniorV ${ }^{\mathrm{V}}$ inícius Herold Dornelas e Silva $^{\mathrm{VI}}$ Tarcízio Antônio Rego de Paula ${ }^{\mathrm{VII}}$ Vanner Boere ${ }^{\mathrm{VIII}}$
\end{abstract}

\section{ABSTRACT}

This paper aims to describe the hematological and biochemical values of wild hybrid marmoset (Callithrix penicillata and C. geoffroyi) found in a forest zone of Southeastern Brazil. The marmosets were anaesthetized using ketamine and xylazine hydrochloride. Blood samples $(0.5-1 \mathrm{~mL})$ were collected through the venipuncture of the femoral vein. Hematological and biochemical analyses were performed using automated counters and biochemical kits. The comparison for sex (adult males vs. adult females) and age class (juvenile vs. adult) physiological data and weight were analyzed through Student's t-test for independent samples. Significant differences between sex were observed in erythrocytes $(P<0.01)$ and hemoglobin $(P<0.05)$. The present study provides essential baseline information on the normal blood values of wild hybrid marmosets, the data of which are not readily accessible from the existing body of scientific literature on nonhuman primates.

Key words: Callithrix sp., hematological indices, marmoset, serum biochemical parameters.

\section{RESUMO}

Este artigo objetiva descrever os valores hematológicos e bioquímicos de saguis híbridos selvagens (Callithrix penicillata e C. geoffroyi) encontrados em uma zona florestal do Sudeste do Brasil. Os saguis foram anestesiados usando cetamina $e$ hidrocloridrato de xilazina. As amostras de sangue (0,5-1 $\mathrm{mL}$ ) foram coletadas por punção da veia femoral. As análises hematológicas e bioquímicas foram realizadas por meio de contadores automáticos e kits bioquímicos. A comparação dos dados fisiológicos e pesos para o gênero (machos vs. fêmeas) e a classe de idade (juvenil vs. adulto) foram analisados através do teste t de Student para amostras independentes. Observaram-se diferenças significativas de gênero para eritrócitos $(P<0,01)$ e hemoglobina $(P<0,05)$. O presente estudo fornece informação básica essencial sobre os valores hematológicos normais de saguis híbridos selvagens, dados que não são prontamente disponíveis na literatura científica atual sobre primatas não humanos.

Palavras-chave: Callithrix sp., índices hematológicos, sagui, parâmetros bioquímicos sorológicos.

\section{INTRODUCTION}

Hybridism is a natural or human-induced phenomenon in animals. Minas Gerais is a state in Brazil with two principal big biomes: Atlantic Forest and Cerrado. In Cerrado, the Brazilian savannah, there is only one species of marmoset, the $\boldsymbol{C}$. penicillata (black tufted marmoset). The more diverse biotope of Atlantic Forest has four species, namely, C. flaviceps, C. geoffroyi, C. aurita, and C. kuhlii (RYLANDS \& FARIA, 1993). However, there was a common and illegal introduction of $\boldsymbol{C}$. jacchus (common marmoset) or $\boldsymbol{C}$. penicillata in many cities of Minas Gerais, and this species commonly occurs in urban areas of the state (GOULART et al., 2010).

Preliminary observations and data of the scientific literature suggest an increasing occurrence

\footnotetext{
'Departamento de Biologia Animal, Universidade Federal de Viçosa (UFV), Viçosa, MG, Brasil.

IPrograma de Pós-graduação em Biologia Animal, UFV, Viçosa, MG, Brasil.

IIIPrograma de Pós-graduação em Biologia Vegetal, Universidade Federal de Minas Gerais (UFMG), Belo Horizonte, MG, Brasil.

IV Universidade Federal de Santa Catarina (UFSC), Curitibanos, SC, Brasil.

'Universidade Vila Velha (UVV), Vila Velha, ES, Brasil.

VIPrograma de Pós-graduação em Veterinária, UFV, Viçosa, MG, Brasil.

VIIDepartamento de Veterinária, UFV, Viçosa, MG, Brasil.

VIIIDepartamento de Bioquímica e Biologia Molecular, UFV, 36570 000, Viçosa, MG, Brasil. E-mail: vanner.boere@ufv.br. Autor para correspondência. 
of hybridism in many places of Brazil. Most primate populations of the Atlantic Forest are restricted to small fragments and that natural populations, particularly marmosets, are threatened by humanintroduced marmosets (PEREIRA et al., 2008).

Despite the hybridism phenomena, there is no information available on the hematology and blood chemistry of the wild hybrids of marmoset in scientific literature. The capture, contention, and breeding of wild marmosets in nature is not easy, and a few researchers are doing this. After that, as described by MCNEES et al. (1984), the small size of the marmoset limits the amount of blood obtained, and the techniques existing in most of the laboratories until today require a bigger amount of blood for analysis.

The hematological and blood biochemical values of free-ranging animals can help in the diagnosis of some diseases, especially when other factors are taken into account that may influence these values like gender, age, stress, or anesthetic agents (FLAIBAN et al., 2008).

This paper aims to describe hematological and biochemical values of wild hybrid marmoset ( $\boldsymbol{C}$. penicillata and $C$. geoffroyi) groups found in the Atlantic Forest fragmented area in Minas Gerais, Southeastern Brazil.

\section{MATERIAL AND METHODS}

Thirty-three hybrids, male and female of black-tufted marmoset ( $\boldsymbol{C}$. penicillata) and Geoffroyi marmoset (C. geoffroyi), from five different groups were used in this work. The age classes were defined according to YAMAMOTO (1993): juvenile animals between five and ten months old and adult above 11 months old. No animal showed signs of clinical disorders or pregnancy during the study period. Marmosets were measured and weight. The body measurements (body and tail) were applied according to the methods described in NAGORSEN and PETERSON (1980). Marmosets were captured at the Biology Forest (20 $35^{\circ}$ ' to $28^{\circ} 50^{\prime}$ 'S and $42^{\circ} 45^{\prime}$ to $43^{\circ} 00^{\prime} \mathrm{W}$ ) from March 2010 to February 2012. This area comprises a fragmented semideciduous submontane forest and has a size of 75 ha. inside the campus of the Federal University of Viçosa (UFV). All groups had some human contact level, so they were habituated to human presence.

All protocols and procedures used were reviewed and approved by the Ethic Committee in Animal Use/UFV (number: 89/2011) and SISBIO/ ICMBio (number: 28632-1). The marmosets were captured with a multiple-entrance trap and anaesthetized using intramuscularly ketamine hydrochloride (Vetaset,
Fort Dodge, Kansas, USA) $10 \mathrm{mg} \mathrm{kg}^{-1}$ body weight with xylazine hydrochloride (Anasedan, Divisão Vetbrands Saúde Animal, SP, BR) $0.5 \mathrm{mg} \mathrm{kg}^{-1}$. Weight was estimated by an experienced primatologist (V.B.) looking the general size of the animals before the anesthesia. Unconscious marmosets were weighed and marked with color stain on body parts. Physical examination was carried out soon after the injection of the anesthesia. Blood samples $(0.5-1 \mathrm{~mL})$ were collected through the venipuncture of the femoral vein. Half of each blood sample was placed into devices containing EDTA (K3EDTA; Labor Import, São Paulo, Brazil) for hematological analysis and the other half into devices without EDTA (Labor Import, São Paulo, Brazil) for biochemical analysis.

After collecting the biological data, the marmosets were kept in a warm $\left(25\right.$ to $\left.28^{\circ} \mathrm{C}\right)$ and darkened room until completely recovered from anesthesia. Four hours after, the animals were released in the same location where they were captured.

After collection, blood samples were sent to the veterinary clinical pathology laboratory of UFV for testing, where the hematological parameters were performed, using an automatic impedance hematology instrument (HumaCount Plus; Human Gesellschaft für Biochemica and Diagnostica MBH, Wiesbaden, Germany). Smears of blood were stained with Wright-Giemsa stain (Newprov, Paraná, Brazil) to differential leukocyte counts. The serum biochemistry parameters: glucose, urea nitrogen, creatinine, alanine aminotransferase (ALT), aspartate aminotransferase (AST), total protein, albumin, globulin, triglycerides, cholesterol, calcium, phosphorus (In vitro, Minas Gerais, Brazil), sodium, and potassium (CELM, São Paulo, Brazil) were performed using an automated clinical chemistry analyzer (HumaStar 300; Human Gesellschaft für Biochemica and Diagnostica MBH, Wiesbaden, Germany). Sodium and potassium concentration was analyzed by a flame photometer (MicroNal - B462, São Paulo, Brazil).

The comparison for sex (adult males vs. adult females) and age class (juvenile vs. adult) physiological data were analyzed through Student's t-test for independent samples. The alpha was $\leq 0.05$ with two-tail distribution.

\section{RESULTS}

The primary goal of these studies was to establish physiological range of hematology and clinical chemistry values in hybrids of Callithrix. This is the first research that describes the globulin values in blood of marmosets. The average values of hematological (red and white cells) data are represented in tables 1 to 4 for sex and age. Significant differences 
Table 1 - Hematology values, (mean, standard deviation, range and P value), without white cells, for male and female hybrids of Callithrix sp.

\begin{tabular}{|c|c|c|c|c|c|c|}
\hline Parameter (unit) & Genera & $\mathrm{N}$ & Mean & $\mathrm{SD}( \pm)$ & Range & $\mathrm{P} \leq$ \\
\hline \multirow{2}{*}{ Erythrocytes $\left(10^{12} 1^{-1}\right)$} & male & 14 & 7.46 & 0.51 & $6.48-8.28$ & \multirow{2}{*}{$0.009 *$} \\
\hline & female & 17 & 6.9 & 0.61 & $5.84-8.12$ & \\
\hline \multirow{2}{*}{ Hemoglobin $\left(\mathrm{g} \mathrm{dl}^{-1}\right)$} & male & 14 & 14.66 & 0.81 & $13.2-15.8$ & \multirow{2}{*}{$0.028 *$} \\
\hline & female & 17 & 13.75 & 1.34 & $10.9-16$ & \\
\hline \multirow{2}{*}{ Hematocrit $\left(11^{-1}\right)$} & male & 16 & 0.46 & 0.06 & $0.27-0.53$ & \multirow{2}{*}{0.35} \\
\hline & female & 17 & 0.44 & 0.05 & $0.36-0.54$ & \\
\hline \multirow{2}{*}{ Total Protein $\left(\mathrm{g} \mathrm{l}^{-1}\right)$} & male & 16 & 72.43 & 5.12 & $66-84$ & \multirow{2}{*}{0.42} \\
\hline & female & 16 & 71.06 & 4.49 & $64-82$ & \\
\hline \multirow{2}{*}{$\operatorname{MCV}(\mathrm{fl})$} & male & 13 & 65.61 & 3.37 & $60-71$ & \multirow{2}{*}{0.95} \\
\hline & female & 17 & 65.55 & 3.05 & $60-72$ & \\
\hline \multirow{2}{*}{$\mathrm{MCH}(\mathrm{pg})$} & male & 12 & 19.45 & 1.28 & $17.3-21.9$ & \multirow{2}{*}{0.48} \\
\hline & female & 17 & 19.81 & 1.35 & $16.2-21.6$ & \\
\hline \multirow{2}{*}{$\operatorname{MCHC}\left(\mathrm{g} \mathrm{dl}^{-1}\right)$} & male & 13 & 30.14 & 1.91 & $25.5-33.04$ & \multirow{2}{*}{0.81} \\
\hline & female & 17 & 30.29 & 1 & $28-32$ & \\
\hline
\end{tabular}

in sex were observed in erythrocytes counts $(\mathrm{P}=0.009)$ and hemoglobin concentration $(\mathrm{P}=0.028)$. Significant age difference was observed only for band neutrophils percentage value $(\mathrm{P}=0.009)$. The data (mean $\pm \mathrm{SD}$ and range) of blood biochemistry are presented in table 5 for sex. It was not observed statistic difference between adult and juvenile for glucose and serum urea nitrogen (Table 6).

Table 2 - White cells values, (mean, standard deviation, range and $\mathrm{P}$ value) for male and female hybrids of Callithrix sp.

\begin{tabular}{|c|c|c|c|c|c|c|}
\hline \multirow{2}{*}{ Basophils (\%) } & male & 16 & 0.00 & 0.00 & $0-0$ & \multirow{2}{*}{0.33} \\
\hline & female & 16 & 0.62 & 0.25 & $0-1$ & \\
\hline \multirow{2}{*}{ Basophils $\left(10^{9} 1-^{1}\right)$} & male & 16 & 0.00 & 0.00 & $0-0$ & \multirow{2}{*}{0.32} \\
\hline & female & 16 & 0.00 & 0.00 & $0-0$ & \\
\hline \multirow{2}{*}{ Eosinophils (\%) } & male & 16 & 1.8 & 3.78 & $0-15$ & \multirow{2}{*}{0.77} \\
\hline & female & 16 & 1.5 & 1.59 & $0-5$ & \\
\hline \multirow{2}{*}{ Eosinophils $\left(10^{9} 1^{-1}\right)$} & male & 16 & 0.08 & 0.21 & $0-0.88$ & \multirow{2}{*}{0.57} \\
\hline & female & 16 & 0.05 & 0.06 & $0-0.23$ & \\
\hline \multirow{2}{*}{ Band neutrophils (\%) } & male & 16 & 0.4 & 0.82 & $0-3$ & \multirow{2}{*}{0.63} \\
\hline & female & 16 & 0.27 & 0.57 & $0-2$ & \\
\hline \multirow{2}{*}{ Band neutrophils $\left(10^{9} 1^{-1}\right)$} & male & 16 & 0.01 & 0.02 & $0-0.05$ & \multirow{2}{*}{0.47} \\
\hline & female & 16 & 0.00 & 0.01 & $0-0.06$ & \\
\hline \multirow{2}{*}{ Segmented $(\%)$} & male & 16 & 48.66 & 12.37 & $27-73$ & \multirow{2}{*}{0.26} \\
\hline & female & 16 & 38.88 & 11.27 & $18-66$ & \\
\hline \multirow{2}{*}{ Segmented $\left(10^{9} 1^{-1}\right)$} & male & 16 & 1.79 & 0.69 & $0.90-3.43$ & \multirow{2}{*}{0.12} \\
\hline & female & 16 & 1.44 & 0.54 & $0.62-2.50$ & \\
\hline \multirow{2}{*}{ Lymphocytes (\%) } & male & 16 & 46.33 & 13.26 & $22-72$ & \multirow{2}{*}{0.33} \\
\hline & female & 16 & 55.94 & 10.92 & $33-77$ & \\
\hline \multirow{2}{*}{ Lymphocytes $\left(10^{9} 1^{-1}\right)$} & male & 16 & 1.87 & 0.87 & 0.543 .72 & \multirow{2}{*}{0.67} \\
\hline & female & 16 & 2.00 & 0.89 & $1.09-3.69$ & \\
\hline \multirow{2}{*}{ Monocytes (\%) } & male & 16 & 2.66 & 2.66 & $0-8$ & \multirow{2}{*}{0.67} \\
\hline & female & 16 & 3.05 & 2.62 & $0-8$ & \\
\hline \multirow{2}{*}{ Monocytes $\left(10^{9} 1^{-1}\right)$} & male & 16 & 0.09 & 0.09 & $0-0.31$ & \multirow{2}{*}{0.56} \\
\hline & female & 16 & 0.11 & 0.10 & $0-0.37$ & \\
\hline \multirow{2}{*}{ Leukocytes $\left(10^{9} 1^{-1}\right)$} & male & 16 & 3.86 & 1.31 & $1.80-7.30$ & \multirow{2}{*}{0.59} \\
\hline & female & 16 & 3.63 & 1.05 & $2.40-6.20$ & \\
\hline \multirow{2}{*}{ Platelet count $\left(10^{3} \mathrm{~mm}^{-3}\right)$} & male & 16 & 404.25 & 179.65 & $152-964$ & \multirow{2}{*}{0.25} \\
\hline & female & 17 & 345.00 & 108.81 & $120-538$ & \\
\hline
\end{tabular}


Table 3 - Hematology values (mean, standard deviation, range and P value), without white cells, for adult and juvenile hybrids of Callithrix sp.

\begin{tabular}{|c|c|c|c|c|c|c|}
\hline Parameter (unit) & Age & $\mathrm{n}$ & Mean & $\mathrm{SD}( \pm)$ & Range & $\mathrm{P} \leq$ \\
\hline \multirow{2}{*}{ Erythrocytes $\left(10^{12} 1^{-1}\right)$} & adult & 24 & 7.26 & 0.60 & $5.93-8.28$ & \multirow{2}{*}{0.11} \\
\hline & juvenile & 7 & 6.8 & 0.62 & $5.84-7.7$ & \\
\hline \multirow{2}{*}{ Hemoglobin $\left(\mathrm{g} \mathrm{dl}^{-1}\right)$} & adult & 24 & 14.29 & 1.15 & $11.8-16$ & \multirow{2}{*}{0.34} \\
\hline & juvenile & 7 & 13.71 & 1.4 & $10.9-15.22$ & \\
\hline \multirow{2}{*}{ Hematocrit $\left(11^{-1}\right)$} & adult & 25 & 0.45 & 0.06 & $0.27-0.54$ & \multirow{2}{*}{0.45} \\
\hline & juvenile & 9 & 0.44 & 0.04 & $0.37-0.50$ & \\
\hline \multirow{2}{*}{ Total Protein $\left(\mathrm{g}^{-1}\right)$} & adult & 24 & 71.83 & 4.79 & $64-84$ & \multirow{2}{*}{0.86} \\
\hline & juvenile & 8 & 71.50 & 5.09 & $66-82$ & \\
\hline \multirow{2}{*}{$\operatorname{MCV}(\mathrm{fl})$} & adult & 23 & 65.36 & 3.34 & $60-72$ & \multirow{2}{*}{0.43} \\
\hline & juvenile & 7 & 65.28 & 2.42 & $64-71$ & \\
\hline \multirow{2}{*}{$\mathrm{MCH}(\mathrm{pg})$} & adult & 22 & 19.5 & 1.34 & $16.2-21.9$ & \multirow{2}{*}{0.22} \\
\hline & juvenile & 7 & 20.17 & 1.15 & $18.6-21.6$ & \\
\hline \multirow{2}{*}{$\operatorname{MCHC}\left(\mathrm{g} \mathrm{dl}^{-1}\right)$} & adult & 23 & 30.22 & 1.49 & $25.5-33.04$ & \multirow{2}{*}{0.95} \\
\hline & juvenile & 7 & 30.25 & 1.35 & $28-32$ & \\
\hline
\end{tabular}

\section{DISCUSSION}

Hematological investigations are an important aid for clinical diagnosis to monitor the health status of wild animals (HAWKEY et al., 1982;
LIDDIE et al., 2010). The physiological status of captive animals could differ from the wild animals for technical reasons (capture method, type of anesthesia, time of blood collection) and for ecologic reasons related to survival under natural conditions (MOEN

Table 4 - White cells values (mean, standard deviation, range and P value) for adult and juvenile hybrids of Callithrix sp.

\begin{tabular}{|c|c|c|c|c|c|c|}
\hline \multirow{2}{*}{ Basophils (\%) } & adult & 25 & 0.41 & 0.20 & $0-1$ & \multirow{2}{*}{0.32} \\
\hline & juvenile & 7 & 0.00 & 0.00 & $0-0$ & \\
\hline \multirow{2}{*}{ Basophils $\left(10^{9} 1^{-1}\right)$} & adult & 25 & 0.00 & 0.00 & $0-0.03$ & \multirow{2}{*}{0.60} \\
\hline & juvenile & 7 & 0.00 & 0.00 & $0-0$ & \\
\hline \multirow{2}{*}{ Eosinophils (\%) } & adult & 25 & 1.58 & 3.11 & $0-15$ & \multirow{2}{*}{0.75} \\
\hline & juvenile & 7 & 1.85 & 1.57 & $0-5$ & \\
\hline \multirow{2}{*}{ Eosinophils $\left(10^{9} 1^{-1}\right)$} & adult & 25 & 0.06 & 0.17 & $0-0.88$ & \multirow{2}{*}{0.98} \\
\hline & juvenile & 7 & 0.06 & 0.08 & $0-0.23$ & \\
\hline \multirow{2}{*}{ Band neutrophils (\%) } & adult & 25 & 0.42 & 0.75 & $0-3$ & \multirow{2}{*}{$0.009 *$} \\
\hline & juvenile & 7 & 0.00 & 0.00 & $0-0$ & \\
\hline \multirow{2}{*}{ Band neutrophils $\left(10^{9} 1^{-1}\right)$} & adult & 25 & 0.01 & 0.02 & $0-0.06$ & \multirow{2}{*}{0.17} \\
\hline & juvenile & 7 & 0.00 & 0.00 & $0-0$ & \\
\hline \multirow{2}{*}{ Segmented (\%) } & adult & 25 & 44.73 & 13.21 & $18-73$ & \multirow{2}{*}{0.14} \\
\hline & juvenile & 7 & 38.14 & 8.9 & $26-49$ & \\
\hline \multirow{2}{*}{ Segmented $\left(10^{9} 1^{-1}\right)$} & adult & 25 & 1.67 & 0.66 & $0.62-3.43$ & \multirow{2}{*}{0.31} \\
\hline & juvenile & 7 & 1.39 & 0.52 & $0.90-2.35$ & \\
\hline \multirow{2}{*}{ Lymphocytes (\%) } & adult & 25 & 50.5 & 13.45 & $22-77$ & \multirow{2}{*}{0.28} \\
\hline & juvenile & 7 & 55.57 & 9.84 & $42-72$ & \\
\hline \multirow{2}{*}{ Lymphocytes $\left(10^{9} 1^{-1}\right)$} & adult & 25 & 1.80 & 0.82 & $0.54-3.72$ & \multirow{2}{*}{0.10} \\
\hline & juvenile & 7 & 2.41 & 0.90 & & \\
\hline \multirow{2}{*}{ Monocytes (\%) } & adult & 25 & 2.5 & 2.21 & $0-8$ & \multirow{2}{*}{0.24} \\
\hline & juvenile & 7 & 4.2 & 3.59 & $0-8$ & \\
\hline \multirow{2}{*}{ Monocytes $\left(10^{9} 1^{-1}\right)$} & adult & 25 & 0.09 & 0.09 & $0-0.37$ & \multirow{2}{*}{0.22} \\
\hline & juvenile & 7 & 0.14 & 0.11 & $0-0.31$ & \\
\hline \multirow{2}{*}{ Leukocytes $\left(10^{9} 1^{-1}\right)$} & adult & 25 & 3.66 & 1.17 & $1.80-7.30$ & \multirow{2}{*}{0.46} \\
\hline & juvenile & 7 & 4.04 & 1.22 & $2.40-6.20$ & \\
\hline \multirow{2}{*}{ Platelet count $\left(10^{3} \mathrm{~mm}^{-3}\right)$} & adult & 25 & 366.00 & 164.89 & $120-964$ & \multirow{2}{*}{0.60} \\
\hline & juvenile & 7 & 397.87 & 79.10 & $321-538$ & \\
\hline
\end{tabular}


Table 5 - Blood biochemistry values (mean, standard deviation, range and P value) for male and female hybrids of Callithrix sp.

\begin{tabular}{|c|c|c|c|c|c|c|}
\hline Parameter (unit) & Genera & $\mathrm{N}$ & Mean & $\mathrm{SD}( \pm)$ & Range & $\mathrm{P} \leq$ \\
\hline \multirow{2}{*}{ Glucose $\left(\mathrm{mmol} \mathrm{l}^{-1}\right)$} & male & 8 & 15.63 & 8.00 & $5.60-26.64$ & \multirow{2}{*}{0.54} \\
\hline & female & 7 & 13.40 & 5.52 & $6.71-25.69$ & \\
\hline \multirow{2}{*}{ Serum urea nitrogen $\left(\mathrm{mmol} \mathrm{l}^{-1}\right)$} & male & 8 & 6.25 & 4.05 & $2.24-15.43$ & \multirow{2}{*}{0.75} \\
\hline & female & 10 & 5.83 & 1.09 & $3.65-6.97$ & \\
\hline \multirow{2}{*}{ Creatinine $\left(\mu \mathrm{mol} \mathrm{l}^{-1}\right)$} & male & 7 & 47.98 & 16.81 & $26.52-70.72$ & \multirow{2}{*}{0.46} \\
\hline & female & 8 & 43.09 & 7.37 & $35.36-53.04$ & \\
\hline \multirow{2}{*}{ ALT - Alanine aminotransferase $\left(\mu \mathrm{Kat} 1^{-1}\right)$} & male & 4 & 0.15 & 0.04 & $0.08-0.20$ & \multirow{2}{*}{0.41} \\
\hline & female & 6 & 0.10 & 0.10 & $0.01-0.30$ & \\
\hline \multirow{2}{*}{ AST - Aspartate aminotransferase $\left(\mu\right.$ Kat $\left.1^{-1}\right)$} & male & 6 & 1.95 & 0.76 & $0.95-2.92$ & \multirow{2}{*}{0.94} \\
\hline & female & 8 & 1.97 & 0.70 & $1.06-3.34$ & \\
\hline \multirow{2}{*}{ Total protein $\left(\mathrm{g} \mathrm{l}^{-1}\right)$} & male & 5 & 65.00 & 7.17 & $57-73$ & \multirow{2}{*}{0.79} \\
\hline & female & 4 & 66.25 & 6.65 & $58-73$ & \\
\hline \multirow{2}{*}{ Albumin $\left(\mathrm{g} \mathrm{l}^{-1}\right)$} & male & 8 & 44.36 & 4.59 & $41.2-55.4$ & \multirow{2}{*}{0.19} \\
\hline & female & 7 & 47.08 & 2.59 & $42.6-50$ & \\
\hline \multirow{2}{*}{ Globulin $\left(\mathrm{g} \mathrm{dL}^{-1}\right)$} & male & 3 & 2.28 & 0.48 & $1.76-2.71$ & \multirow{2}{*}{0.38} \\
\hline & female & 4 & 1.96 & 0.40 & $1.54-3.38$ & \\
\hline \multirow{2}{*}{ Triglycerides $\left(\mathrm{mmol} \mathrm{l}^{-1}\right)$} & male & 8 & 0.87 & 0.39 & $0.40-1.50$ & \multirow{2}{*}{0.78} \\
\hline & female & 7 & 0.93 & 0.41 & $0.64-1.54$ & \\
\hline \multirow{2}{*}{ Cholesterol $\left(\mathrm{mmol} \mathrm{l}^{-1}\right)$} & male & 8 & 3.73 & 0.74 & $2.53-4.89$ & \multirow{2}{*}{0.52} \\
\hline & female & 7 & 3.44 & 0.95 & $2.84-5.18$ & \\
\hline \multirow{2}{*}{ Calcium $\left(\mathrm{mmol} \mathrm{l}^{-1}\right)$} & male & 3 & 5.16 & 0.58 & $4.50-5.40$ & \multirow{2}{*}{0.45} \\
\hline & female & 4 & 4.71 & 0.81 & $3.50-6.60$ & \\
\hline \multirow{2}{*}{ Phosphorus (mmol $1^{-1}$ ) } & male & 3 & 0.95 & 0.68 & $0.51-0.74$ & \multirow{2}{*}{0.35} \\
\hline & female & 4 & 0.61 & 0.11 & $0.51-1.74$ & \\
\hline \multirow{2}{*}{ Sodium $\left(\mathrm{mmol} \mathrm{l}^{-1}\right)$} & male & 5 & 143.80 & 12.96 & $122-156$ & \multirow{2}{*}{0.42} \\
\hline & female & 4 & 149.50 & 3.69 & $145-153$ & \\
\hline \multirow{2}{*}{ Potassium $\left(\mathrm{mmol} \mathrm{l}^{-1}\right)$} & male & 5 & 5.04 & 2.74 & $3.7-9.8$ & \multirow{2}{*}{0.85} \\
\hline & female & 4 & 4.77 & 1.20 & $3.7-6.5$ & \\
\hline
\end{tabular}

et al., 2010). To the best of our knowledge, there are no published hematologic and blood biochemistry values for wild marmosets.

Male and female hybrid wild marmosets have similar hematological profiles, but some important differences have emerged in this investigation. The values of male marmoset erythrocytes and hemoglobin concentration were higher than that of females. Erythrocytes are cells carrying the hemoglobin, protein carrying oxygen to cells and carbon dioxide from cells to lungs. Due to the testosterone hormone effect, the hematopoietic tissue like the bone marrow of males is more stimulated in comparison to females (RAY et al., 2008). It is a natural and well-known physiological phenomenon in mammals in general, but exceptions occur. For example, in black-tufted marmosets ( $\boldsymbol{C}$. penicillata), there are no hematological differences between males and females (BOERE et al., 2005). However, it is difficult to assign if this difference is an artifact of captivity or a true difference between pure $\boldsymbol{C}$. penicillata and hybrid marmosets.

Age-class differences emerged on band neutrophils number in the present study. It was possible to detect band neutrophils in the blood of adult but not in juvenile marmosets. This contrast between adults and juveniles is likely an artifact of elevated values in three adults. Despite the clinical health appearance, band neutrophils were evident upon laboratory examination, suggesting an inflammation in those three marmosets (WEISER, 2007).

The results obtained in our study are similar to the data found in captive $\boldsymbol{C}$. penicillata, $C$. jacchus, and $\boldsymbol{C}$. geoffroyi for all hematological parameters and for some blood chemistry parameters (BOERE et al., 2005; NASCIMENTO et al., 1993; PETERS et al., 2003; YARBROUGH et al., 1984). On the other hand, glucose and serum urea analysis showed higher values than references. The concentration of glucose in the blood serum increases in marmosets 
Table 6 - Glucose and serum urea nitrogen values (mean, standard deviation, range and P value) for adult and juvenile hybrids of Callithrix sp.

\begin{tabular}{lllllc}
\hline Parameters & Age & N & Mean & SD $( \pm)$ & Range \\
\hline \multirow{2}{*}{ Glucose $\left(\mathrm{mg} \mathrm{dL}^{-1}\right)$} & Adult & 16 & 11.11 & 9.71 & $5.60-26.64$ \\
& Juvenile & 4 & 10.28 & 3.27 & $6.71-21.25$ \\
Serum urea nitrogen $\left(\mathrm{mg} \mathrm{dL}^{-1}\right)$ & Adult & 16 & 5.59 & 3.45 & $3.65-15.43$ \\
& Juvenile & 4 & 4.77 & 1.87 & $2.24-6.72$ \\
\hline
\end{tabular}

due to excitement and stress. Ketamine, which was used in this study to anesthetize the marmosets, can also stimulate the increase of blood glucose (SHIIGI $\&$ CASEY, 2001). The higher serum urea nitrogen in wild marmoset could be due to several causes, including mild dehydration and a diet with higher protein content (i.e., insects and eggs) than the diet of captive marmosets (WEISER, 2007).

Elevated liver enzymes (ALT and AST) may indicate inflammation or damage to cells in the liver. The AST level of wild hybrid marmosets was similar to other marmosets, presuming a normal liver parameter. The ALT was very low in the sample of wild hybrid marmosets in comparison with captive pure marmosets (C. jacchus) (YARBROUGH et al., 1984). Low ALT of wild hybrid marmosets studied here is unclear because both the older and younger marmosets have low ALT levels. If AST is normal and marmosets are healthy, low ALT could be interpreted as just a peculiar physiological characteristic of liver function in wild hybrid marmosets studied here.

In summary, the present study showed some biochemical and hematologic variables in wild hybrid marmosets. Some sex differences emerged from the sample used here. Only band neutrophils numbers are not similar between juveniles and adult marmosets. This study provides essential baseline information on the normal blood values of wild hybrid marmosets, the data of which are not readily accessible from the existing scientific literature on nonhuman primates.

\section{ACKNOWLEDGEMENTS}

F. F. R. Silva, L. F. F., A. O. Tavela, M. Carretta $\mathrm{Jr}$ and V. H. D. Silva were granted by Conselho Nacional de Desenvolvimento Científico e Tecnológico (CNPq) and Coordenação de Aperfeiçoamento de Pessoal de Nível Superior (Capes) during the study.

\section{REFERENCES}

BOERE, V. et al. Comparison between sex and age class on some physiological, thermal, and hematological indices of the cerrado's marmoset (Callithrix penicillata). Journal of Medical Primatology, v.34, p.156-162, 2005. Available from: <http://onlinelibrary.wiley. com/doi/10.1111/j.1600-0684.2005.00101.x/pdf>. Accessed: Dec. 20, 2011. doi: 10.1111/j.1600-0684.2 005.00101.x.

FLAIBAN, K.K.M.C. et al. Valores hematológicos de bugios pretos (Alouatta caraya) de vida livre da região do Alto Rio Paraná, sul do Brasil. Arquivo Brasileiro de Medicina Veterinária e Zootecnia, v.61, p.628-634, 2008. Available from: <http://www. scielo.br/pdf/abmvz/v61n3/16.pdf>. Accessed: Feb. 15, 2012. doi: 10.1590/S0102-09352009000300016.

GOULART, V.D.L.R. et al. Analysis of callouts made in relation to wild urban marmosets (Callithrix penicillata) and their implications for urban species management. European Journal of Wildlife Research, v.56, p.641-649. 2010. Available from: $<$ http://www.springerlink.com/content/g2214k7442pm6777/>. Accessed: Dec. 10, 2011. doi: 10.1007/s10344-009-0362-4.

HAWKEY, C.M. et al. Clinical hematology of the Common Marmoset Callithrix jacchus. American Journal of Primatology, v.3, p.179-199, 1982. Accessed: Oct. 20, 2011. doi: 02752565/82/0301-04-0179\$06.0.

LIDDIE, S. et al. Clinical chemistry and hematology values in a Caribbean population of African green monkeys. Journal of Medical Primatology, v.39, p.389-398, 2010. Available from: $<$ http://onlinelibrary.wiley.com/doi/10.1111/j.1600-0684.201 0.00422.x/full>. Accessed: Dec. 11, 2011. doi:10.1111/j.1600- 06 84.2010.00422.x.

MCNEES, D.W. et al. Blood chemistry of the common marmoset (Callithrix jacchus) maintained in an indoor-outdoor environment: Primate comparisons. Primates, v.25, p.103-109, 1984. Available from: <http://www.springerlink.com/content/ v1416nh4607v38t2/>. Accessed: Nov. 05, 2011. doi: 10.1007/ BF02382300

MOEN, R. et al. Hematology, serum chemistry, and body mass of free-ranging and captive Canada lynx in Minnesota. Journal of Wildlife Diseases, v.46, p.13-22, 2010. Available from: <http:// www.jwildlifedis.org/content/46/1/13.full.pdf $>$. Accessed: Jan. 20,2012

NAGORSEN, D.W.; PETERSON, R.L. Mammal collectors manual. Toronto: The Royal Ontario Museum, 1980. 79p.

NASCIMENTO, M.D et al. Hematological profiles of Callithrix geoffroyi, Callithrix kuhli and Callithrix aurita (CallitrichidaePrimate). In: YAMAMOTO, M.E.; SOUZA, M.B.C. (Eds.). A primatologia no Brasil 4. Natal: UFRN, 1993. P.227-243.

PEREIRA, D.G. et al. Interação entre calitriquídeos exóticos e nativos no Parque Nacional da Serra dos Órgãos-RJ. Espaço \& Geografia, v.11, p.87-114, 2008. Available from: <http://www. 1sie.unb.br/espacoegeografia/index.php/espacoegeografia/article/ view/80/79>. Accessed: Jun. 17, 2011. 
PETERS, V.M. et al. Callithrix jacchus penicillata blood count. Boletim do Centro de Biologia Reprodutiva, v.22, p.101-104, 2003.

RAY, R. et al. Sex steroids and stem cell function. Journal of Molecular Medicine, v.14, p.493-501, 2008. Available from: $<$ http://www.ncbi.nlm.nih.gov/pmc/articles/PMC2376641/>. Accessed: Fev. 02, 2012. doi: 10.2119/2008-00004.Ray.

RYLANDS, A.B.; FARIA, D.S. Habitats, feeding ecology, and home range size in the genus Callithrix. In: RYLANDS, A.B. (Ed.). Marmosets and tamarins: systematics, behavior and ecology. Oxford: Oxford University, 1993. p.262-272.

SHIIGI, Y.; CASEY, D.E. Effects of benztropine on ketamine-induced behaviors in Cebus monkeys. Behavioral
Pharmacology, v.12, p.293-298, 2001. Available from: <http:// journals.1ww.com/behaviouralpharm/pages/default.aspx $>$. Accessed: May. 15, 2011.

YAMAMOTO, M.E. From dependence to sexual maturity: the behavioural ontogeny of Callitrichidae. In: RYLANDS, A.B, (Ed.). Marmosets and tamarins: systematics, ecology and behaviour. Oxford: Oxford University, 1993. p.235-254.

YARBROUGH, L.W. et al. Serum biochemical, hematological and body measurement data for common marmosets (Callithrix jacchus jacchus). Laboratory Animal Science, v.34, p.276-80, 1984.

WEISER, G. Interpretação da resposta leucocitária nas doenças. In: THRALL, M.A. (Ed.). Hematologia e bioquímica clínica. São Paulo: Rocca, 2007. p.127-141. 